\title{
Precipitating Factors for Delirium in Hospitalized Elderly Persons
}

\section{Citation}

Inouye, Sharon K, and Peter A. Charpentier. 1996. Precipitating Factors for Delirium in Hospitalized Elderly Persons. JAMA 275, no. 11: 852. doi:10.1001/jama.1996.03530350034031.

\section{Published Version}

doi:10.1001/jama.1996.03530350034031

\section{Permanent link}

http://nrs.harvard.edu/urn-3:HUL.InstRepos:33751440

\section{Terms of Use}

This article was downloaded from Harvard University's DASH repository, and is made available under the terms and conditions applicable to Other Posted Material, as set forth at http:// nrs.harvard.edu/urn-3:HUL.InstRepos:dash.current.terms-of-use\#LAA

\section{Share Your Story}

The Harvard community has made this article openly available.

Please share how this access benefits you. Submit a story.

Accessibility 


\title{
Precipitating Factors for Delirium in Hospitalized Elderly Persons
}

\section{Predictive Model and Interrelationship With Baseline Vulnerability}

\author{
Sharon K. Inouye, MD, MPH; Peter A. Charpentier, MPH
}

Objectives.-To prospectively develop and validate a predictive model for delirium based on precipitating factors during hospitalization, and to examine the interrelationship of precipitating factors and baseline vulnerability.

Design.-Two prospective cohort studies, in tandem.

Setting.-General medical wards, university teaching hospital.

Patients.-For the development cohort, 196 patients aged 70 years and older with no delirium at baseline, and for the validation cohort, 312 comparable patients.

Main Outcome Measure.-New-onset delirium by hospital day 9, defined by the Confusion Assessment Method diagnostic criteria.

Results.-Delirium developed in 35 patients (18\%) in the development cohort. Five independent precipitating factors for delirium were identified: use of physical restraints (adjusted relative risk [RR], 4.4; 95\% confidence interval [Cl], 2.5 to 7.9 ), malnutrition (RR, $4.0 ; 95 \% \mathrm{Cl}, 2.2$ to 7.4 ), more than three medications added (RR, $2.9 ; 95 \% \mathrm{Cl}, 1.6$ to 5.4 ), use of bladder catheter (RR, $2.4 ; 95 \% \mathrm{Cl}, 1.2$ to 4.7 ), and any iatrogenic event (RR, $1.9 ; 95 \% \mathrm{Cl}, 1.1$ to 3.2 ). Each precipitating factor preceded the onset of delirium by more than 24 hours. A risk stratification system was developed by adding 1 point for each factor present. Rates of delirium for lowrisk (0 points), intermediate-risk ( 1 to 2 points), and high-risk groups ( $\geq 3$ points) were $3 \%, 20 \%$, and $59 \%$, respectively $(P<.001)$. The corresponding rates in the validation cohort, in which 47 patients (15\%) developed delirium, were $4 \%, 20 \%$, and $35 \%$, respectively $(P<.001)$. When precipitating and baseline factors were analyzed in cross-stratified format, delirium rates increased progressively from low-risk to high-risk groups in all directions (double-gradient phenomenon). The contributions of baseline and precipitating factors were documented to be independent and statistically significant.

Conclusions.-A simple predictive model based on the presence of five precipitating factors can be used to identify elderly medical patients at high risk for delirium. Precipitating and baseline vulnerability factors are highly interrelated and contribute to delirium in independent, substantive, and cumulative ways.

(JAMA. 1996;275:852-857)

DELIRIUM, an acute disorder of attention and cognition, has become increasingly recognized as a common and serious problem for hospitalized elderly patients. Delirium occurs in $14 \%$ to $56 \%$ of elderly hospitalized patients with associated hospital mortality rates of $10 \%$ to $65 \%$, longer, costlier hospitalizations, and increased rates of nursing home placement. ${ }^{1}$ Development of effective

\footnotetext{
From the Departments of Internal Medicine (Dr Inouye) and Epidemiology and Public Health (Mr Charpentier), Yale University School of Medicine, New Haven, Conn.

Reprint requests to Yale University School of Medicine, Yale-New Haven Hospital, 20 York St, Tompkins 15. New Haven, CT 06504 (Dr Inouye)
}

strategies to prevent this serious problem requires an in-depth understanding of risk factors for delirium.

To date, 10 prospective studies ${ }^{2-11}$ have been published that systematically examine risk factors for delirium in hospitalized elderly patients. These studies examine baseline vulnerability and precipitating factors simultaneously, which does not allow for analyzing the differential effects of precipitating factors in patients with dissimilar baseline vulnerabilities. Baseline vulnerability factors are defined as predisposing risk factors for delirium present at the time of hospital admission. Precipitating factors are defined as noxious insults or hospitalization-related factors that contribute to delirium. To our knowledge, no previous studies have examined the interrelationship of baselire vulnerability and precipitating factors for delirium.

The underlying hypothesis behind the current study is that delirium is rarely caused by a single factor; rather, it represents an intrinsically multifactorial syndrome-similar to other common geriatric syndromes (eg, falls and incontinence). ${ }^{12}$ We propose a multifactorial model, as shown in Figure 1, in which delirium involves a complex interrelationship between baseline (admission) patient vulnerability and precipitating factors or insults, occurring during hospitalization. Patients who are highly vulnerable to delirium at baseline (eg, cognitively impaired and/or severely ill) may develop delirium with any precipitating factor, even of mild degree. Conversely, patients with low vulnerability would be resistant to the development of delirium, even with noxious insults. We further hypothesize that the effects of baseline and precipitating factors on development of delirium are cumulative.

Our previous work focused on evaluating baseline vulnerability, the first domain of our multifactorial model(Figure 1). In a previous study, ${ }^{13}$ we developed and validated a predictive model that would assess baseline vulnerability to delirium, based on the presence of predisposing factors at hospital admission. The current study builds on the previous work and addresses the precipitating, hospitalization-related factors domain of the model.

The specific objectives of the current study are (1) to identify potential precipitating factors for delirium during hospitalization in general medicine patients, (2) to develop a predictive model for delirium based on these precipitating factors and to validate this model in an independent sample, and (3) to examine the interrelationship of baseline (admission) vulnerability and precipitating factors for delirium. 


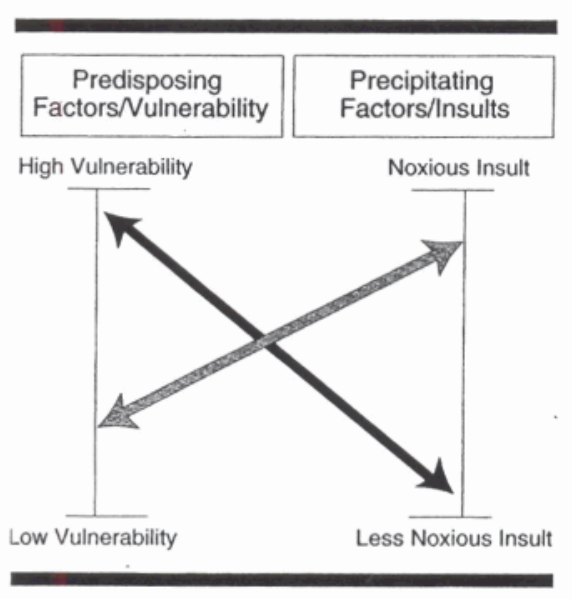

Figure 1.-Multifactorial model of delirium. The development of delirium involves a complex interrelationship between baseline patient vulnerability (line to left) and precipitating factors or insults (line to right). For example, a patient with low vulnerability would require noxious insults to develop delirium (gray arrow). Conversely, a highly vulnerable patient may develop delirium even with relatively trivia insults (black arrow).

\section{METHODS}

\section{Development Study}

Patients.-The potential study participants were 342 patients admitted consecutively during weekdays who were aged 70 years and older, with no evidence of delirium at baseline, admitted to the six general medicine (nonintensive care) floors at Yale-New Haven (Conn) Hospital from November 6, 1989, through June 22, 1990. Yale-New Haven Hospital is an 800-bed urban teaching hospital with 200 medical beds, serving a large community as well as a referral population. Patients were excluded if they could not be interviewed for any reason, ie, intubation, coma, severe aphasia, or terminal condition $(n=61)$; if they were discharged in less than 48 hours $(n=44)$; if the subjects or their physicians declined participation $(n=29)$; if they had been enrolled in the study on a previous admission $(n=1)$; or if risk factor data were missing $(n=1)$. Of the 206 patients enrolled in the study, 10 (5\%) were excluded due to the presence of delirium at the baseline assessment. Thus, the final sample included 196 participants.

Clinical Evaluation.-Trained clinician-researchers carried out structured interviews with the patients and their primary nurses from entry until hospital discharge. The baseline patient interview, completed within 48 hours of admission, included demographic information, current living situation, self-reported activities of daily living 2 weeks before admission, ${ }^{14}$ Mini-Mental State Examination (MMSE) score, ${ }^{15}$ standard near-vision (Jaeger type) and hearing (whisper) tests, ${ }^{16,17}$ the Confusion Assessment Method rating for delirium, ${ }^{18}$ and a standardized skin check for breakdown at 11 pressure points. ${ }^{19}$ Baseline information obtained from the nurses included an overall rating of illness severity. ${ }^{20}$ Early in the hospital stay, family members or caregivers underwent a structured interview that included the modified Blessed Dementia Rating Scale $^{21,22}$ and an estimation of the duration of any cognitive impairment.

Thereafter, the clinician-researchers interviewed the patients and their nurses every other day (eg, total of five interviews during the first 9 days) and reviewed medical records to detect any new cases of delirium. In addition, the patient interviews included a sleep questionnaire (assessing quantity and quality of sleep and level of fatigue) and direct observation-based ratings of use of restraints, presence of immobilizing devices (medical equipment that would limit patients' freedom of movement, eg, intravenous lines, continuous oxygen, or nasogastric tubes on suction), evidence of dehydration (dry mucous membranes), and surveillance for skin breakdown using the standardized skin check. The nurse interviews assessed any evidence of delirium since the last interview, use of restraints or bladder catheter (eg, indwelling Foley or suprapubic catheter), patient's level of mobility and out-of-bed time, presence of dehydration, and use of new skin care for pressure ulcers. Medical records were extracted in detail by experienced, trained nurse researchers for information, including medical diagnoses, medications, laboratory results, vital signs, weights, use of restraints (orders and/or progress notes), and documentation of iatrogenic events, pressure ulcers, or dehydration.

All data were obtained using standardized instruments. The clinician-researchers and medical record abstractors were blinded to the research question and hypotheses. Informed consent was obtained from the patient or, for those with significant cognitive impairment, from the closest relative. This study was approved by the Institutional Review Board of Yale University School of Medicine.

Outcome.-The outcome for this study was new-onset delirium, defined by the Confusion Assessment Method diagnostic criteria, ${ }^{18}$ which requires the presence of acute onset and fluctuating course, inattention, and either disorganized thinking or altered level of consciousness. In a previous validation study, ${ }^{18}$ these criteria had a sensitivity of $94 \%$ to $100 \%$ and a specificity of $90 \%$ to $95 \%$ when compared with the ratings of geropsychiatrists. For this study, Confusion Assessment Method ratings were based on direct observations of the clinicianresearchers at the bedside, which were supplemented by nurse interviews and medical record data. The nurse interviews included information on any mental status change since the prior interview. Using information from all sources (interviewer observation, nurse reports, and medical record), two investigators adjudicated the earliest onset of delirium symptoms in all cases. The delirium date used in these analyses was the date of onset of the first episode of delirium. A case of delirium developing at any time during the period at risk (see "Definition of Variables" section) was included in these analyses; however, a given patient could develop delirium only once (recurrent episodes of delirium were not counted in the analyses).

Definition of Variables.-Baseline risk for delirium was defined according to a previously developed and validated predictive model, described in detail elsewhere. ${ }^{13}$ The predictive model was based on four baseline risk factors for delirium (present at hospital admission): vision impairment (visual acuity $<20 / 70$ ), severe illness (defined as an Acute Physiology and Chronic Health Evaluation II [APACHE II] score $>16^{23}$ or a nurse rating of severe illness ${ }^{20}$ ), cognitive impairment (MMSE score $<24$ ), and a serum urea nitrogen:creatinine ratio of 18 or greater. Risk groups were developed by adding 1 point for each risk factor present, stratified as low-risk (no risk factors), intermediate-risk (one to two factors), and high-risk (three to four factors) groups. Since measures of illness severity and comorbidity were considered previously as baseline or predisposing factors, ${ }^{13}$ these were not evaluated as precipitating factors in the current study.

To ensure temporal precedence (ie, that the precipitating factor preceded the delirium), we required that each potential precipitating factor be present for at least 24 hours before the onset of delirium. In addition, for all potential precipitating factors variables, the "at-risk" or exposure period was truncated at hospital day 9 -to create comparable at-risk periods between delirium and nondelirium groups, as well as to minimize the effect of long hospitalizations. The risk of delirium decreased greatly after hospital day 9 . By day 9,35 ( $88 \%)$ of 40 delirium cases had developed delirium and 122 (78\%) of 156 nondelirium cases had been discharged.

The "more than three medications added" variable is defined as the addition of more than three medication types during the period from 48 to 24 hours before the onset of the delirium. This variable was calculated based on change in the count of total number of types of medications received by the patient each day. "Prolonged emergency department 
stay" was identified when more than 12 hours elapsed between the patient's emergency department admission time and arrival time on the hospital ward. "Iatrogenic events" were defined using standard criteria ${ }^{24,25}$ as an illness resulting from a diagnostic procedure or therapeutic intervention or any harmful occurrence that was not a natural consequence of the patient's underlying illness (and not present at hospital admission). These events were categorized into six major groups: (1) cardiopulmonary complications (eg, pulmonary embolism or pulmonary edema due to volume overload); (2) hospital-acquired infections (eg, probable aspiration pneumonia, wound infection/dehiscence, or urinary tract infection following instrumentation); (3) medication-related complications (eg, toxicity or sensitivity); (4) complications of diagnostic or therapeutic procedures (eg, intravenous catheter complication including infection or phlebitis, transfusion reaction, or prolonged bleeding due to procedure and/ or overanticoagulation); (5) unintentional injury (eg, falls, fractures, or lacerations); or (6) other (eg, new pressure sore or fecal impaction).

"Malnutrition" was defined as a serum albumin level less than $30 \mathrm{~g} / \mathrm{L}$ occurring during hospitalization, but preceding the onset of delirium by at least 24 hours. The same time frame was used in all subsequent variables. "Respiratory insufficiency" was defined as a $\mathrm{PCO}_{2}$ greater than $45 \mathrm{~mm} \mathrm{Hg}$ or $\mathrm{PO}_{2}$ less than $50 \mathrm{~mm} \mathrm{Hg}$ or oxygen saturation less than $80 \%$. "Intercurrent infection" included any pneumonia, urinary tract infection, or wound infection. "Dehydration" was defined by evidence of dehydration by nurses or trained clinician-researchers at the bedside or by documentation in the medical record.

\section{Validation Study}

Patients.-Patients potentially eligible for the validation study included 801 patients admitted consecutively during the week aged 70 years and older admitted to the medicine service at YaleNew Haven Hospital from July 9, 1990, to July 31,1991 . The inclusion and exclusion criteria were identical to those of the development cohort. Patients were excluded if they could not be interviewed $(n=147)$, if they were discharged in less than 48 hours $(n=118)$, if the subjects or their physicians declined participation $(n=56)$, if they had been enrolled in the study on a previous admission $(n=100)$, or for other reasons, such as respiratory isolation $(n=61)$. Of the 319 patients enrolled in the study, seven $(2 \%)$ were excluded due to the presence of delirium at the baseline assessment. Thus, the final sample included 312 participants.

Procedure.-The clinical evaluation, outcome, and definitions of variables were identical to those in the development study. The same trained clinicianresearchers carried out the study using identical data collection instruments, blinded to the research questions.

\section{Statistical Analyses}

For each baseline characteristic, the two cohorts (development and validation) were compared using appropriate test statistics, either $t$ test statistics for continuous variables or $\chi^{2}$ statistics for categorical variables. Crude relative risks (RRs) were calculated as the ratio of number of events (delirium) in the group when the precipitating factor was present compared with that in the group with the precipitating factor absent. Confidence intervals of $95 \%$ were calculated.

The object of the analyses in the development cohort was to identify factors that could be combined into a score predictive of delirium onset. The strategy to select among potential candidate variables included grouping the variables into axes, then reducing the variables on each axis using multivariable binomial regression models. ${ }^{26}$ This modeling technique accounts for the differing times of exposure and differing times to onset of delirium. The binomial (relative risk) model was chosen over the proportional hazards model because it (1) models actual rates (ie, appropriate to the primary outcome and delirium rate), (2) yields direct estimates of $R R$, and (3) does not require fulfillment of the proportionality assumption. Nonetheless, results were similar between the two models (results not shown) and the same final variables would have been selected using either strategy.

Potential precipitating factors for delirium were classified into four axes: immobility, medications, iatrogenic events, and intercurrent illness. An optimal subset of variables from each axis was selected based on fulfillment of three criteria: (1) a priori clinical relevance, (2) RR of 1.5 or greater, and (3) meeting statistical selection criteria by binomial regression models. The models used both forward- and backward-stepping algorithms $(P$ value for entry and removal set to .10). Hospital day, categorized into three groups (days 1 to 3,4 to 6 , and 7 to 9 ), was included in each model to account for the declining risk of delirium over time. Collinearity was assessed by inspecting changes to SEs of parameter estimates as variables were added to or removed from the models. ${ }^{27}$

Using this method, 11 variables were selected as the optimal variables from each axis. These 11 variables were then combined, and the same model-building strategy just described was applied. Because of our a priori assumption that each axis is important in precipitating delirium, we required that at least one factor from each axis be included in the final model. The predictive model created in the development cohort was then tested in the validation cohort.

Adjusted RRs and confidence intervals were calculated from the parameter estimates and standard errors. Overall $\chi^{2}$ and Mantel-Haenszel trend tests were used to compare rates of delirium between risk groups in both cohorts. The degree to which the models fit the observed data was inferred by evaluating the goodness-of-fit $\left(\mathrm{G}^{2}\right)$ statistics from rate models that included the baseline risk score, precipitating factors score, and hospital day ${ }^{28}$ in each cohort.

All analyses were carried out using SAS Version 6(SAS Institute, Cary, NC) or GLIM 3.77 (Numerical Algorithms Group, Oxford, England) software.

\section{RESULTS}

Characteristics of the development cohort are shown in Table 1 . Of the 196 subjects, $35(18 \%)$ had new-onset delirium by hospital day 9 , with a median onset on hospital day 4 (range, 3 to 39 days). The median length of hospital stay for this cohort was 8 days (range, 3 to 67 days).

\section{Development of the Predictive Model}

The 25 candidate variables considered as potential precipitating factors for delirium in the development cohort-divided into the four axes described previously-are shown in Table 2 . From the "immobility" axis (axis 1), four variables met the selection criteria for inclusion in the multivariable model. The composite variable "use of three or more immobilizing devices or restraints" was not included since the "use of physical restraints" variable was more strongly associated with delirium.

From the "medications" axis (axis 2), two variables met the selection criteria for inclusion in the multivariable model. The variable coding for use of "two or more psychoactive medications" was chosen over its component variables because of its higher RR. From the "iatrogenic events" axis (axis 3), two variables met selection criteria. The composite variable "any iatrogenic event" was selected over its component variable "unintentional injury" because of its greater clinical relevance and higher prevalence. From the "intercurrent illness" axis (axis 4), three variables were selected. The "intercurrent infection" variable did not meet our selection criteria.

The 11 variables selected as the optimal variables from each axis were nar- 
Table 1.-Baseline Characteristics of Subjects in Two Cohorts

\begin{tabular}{|c|c|c|}
\hline Characteristics* & $\begin{array}{l}\text { Development Cohort } \\
(n=196)\end{array}$ & $\begin{array}{l}\text { Validation Cohor } \\
(n=312)\end{array}$ \\
\hline Age, y (mean $\pm S D)$ & $78.5 \pm 5.7$ & $78.5 \pm 6.2$ \\
\hline Female, No. (\%) & $112(57)$ & $172(55)$ \\
\hline White, No. (\%) & $178(91)$ & $283(91)$ \\
\hline Education, y $($ mean $\pm S D) \dagger$ & $11.2 \pm 3.4$ & $11.4 \pm 3.5$ \\
\hline Married, No. $(\%) \dagger$ & $84(43)$ & $153(49)$ \\
\hline Living alone, No. (\%) & $85(43) \ddagger$ & $86(28) \ddagger$ \\
\hline Any impairment in activities of daily living, No. (\%) & $9(5)$ & $16(5)$ \\
\hline $\begin{array}{l}\text { Mini-Mental State Examination score } \\
\text { Mean } \pm \text { SD }\end{array}$ & $22.4 \pm 6.0$ & $22.4 \pm 7.2$ \\
\hline Score <24, No. $(\%)$ & $93(47)$ & $128(41)$ \\
\hline APACHE II score, mean \pm SD $\dagger$ & $14.0 \pm 3.7 \ddagger$ & $15.4 \pm 3.5 \ddagger$ \\
\hline $\begin{array}{l}\text { Baseline delirium risk group, №. (\%)§ } \\
\text { Low }\end{array}$ & $22(11)$ & $34(11)$ \\
\hline Intermediate & $1+1(57)$ & $176(56)$ \\
\hline High & $63(32)$ & $102(33)$ \\
\hline
\end{tabular}

Table 2.-Variables Considered as Precipitating Factors for Delirium in the Development Cohort $(n=196)$

\begin{tabular}{|c|c|c|c|}
\hline \multirow[b]{2}{*}{ Factor } & \multicolumn{2}{|c|}{ Delirium When Factor } & \multirow[b]{2}{*}{ RR $(95 \% \mathrm{CI})^{*}$} \\
\hline & $\begin{array}{l}\text { Present, } \\
\text { No. (\%) }\end{array}$ & $\begin{array}{l}\text { Absent, } \\
\text { No. (\%) }\end{array}$ & \\
\hline \multicolumn{4}{|l|}{ Axis 1 , immobility } \\
\hline Use of physical restraints & $14 / 31(45)$ & $21 / 165(13)$ & $3.5(2.0-6.3) \dagger$ \\
\hline Use of bladder catheter & $18 / 50(36)$ & $17 / 146(12)$ & $3.1(1.7-5.5) \dagger$ \\
\hline Use of $\geq 3$ immobilizing devices & $14 / 52(27)$ & $21 / 144(15)$ & $1.8(1.0-3.4) \dagger$ \\
\hline Use of $\geq 3$ immobilizing devices or restraints & $20 / 71(28)$ & $15 / 125(12)$ & $2.3(1.3-4.2)$ \\
\hline Out of bed less than once a day & $21 / 78(27)$ & $14 / 118(12)$ & $2.3(1.2-4.1) \dagger$ \\
\hline \multicolumn{4}{|l|}{ Axis 2 , medications } \\
\hline Anticonvulsants & $3 / 5(60)$ & $32 / 191(17)$ & $3.6(1.3-9.8)$ \\
\hline Major tranquilizers & $2 / 6(33)$ & $33 / 190(17)$ & $1.9(0.5-6.9)$ \\
\hline Antiemetics & $6 / 16(38)$ & $29 / 180(16)$ & $2.3(1.1-5.1)$ \\
\hline Narcotics & 8/31 (26) & $27 / 165(16)$ & $1.6(0.8-3.2)$ \\
\hline$\geq 2$ Psychoactive medications & $5 / 7(71)$ & $30 / 189(16)$ & $4.5(2.1-9.9) \dagger$ \\
\hline Total No. of medications $>6$ & $16 / 91(18)$ & $19 / 105(18)$ & $1.0(0.5-1.8)$ \\
\hline$>3$ Medications added & $10 / 18(56)$ & $25 / 178(14)$ & $4.0(2.1-7.3) \dagger$ \\
\hline \multicolumn{4}{|l|}{ Axis 3 , iatrogenic events } \\
\hline Prolonged emergency department stay ( $>12 \mathrm{~h})$ & $16 / 57(28)$ & $19 / 139(14)$ & $2.1(1.1-3.7) \dagger$ \\
\hline Volume overload & $4 / 16(25)$ & $31 / 180(17)$ & $1.5(0.6-3.7)$ \\
\hline Intravenous catheter complications & $7 / 27(26)$ & $28 / 169(17)$ & $1.6(0.7-3.3)$ \\
\hline Prolonged bleeding & $1 / 4(25)$ & $34 / 192(18)$ & $1.4(0.2-8.5)$ \\
\hline Urinary tract infection following instrumentation & $2 / 9(22)$ & $33 / 187(18)$ & $1.3(0.3-4.6)$ \\
\hline Transfusion reaction & $2 / 13(15)$ & $33 / 183(18)$ & $0.9,(0.2-3.1)$ \\
\hline Unintentional injury & $5 / 6(83)$ & $30 / 190(16)$ & $5.3(2.4-11.4)$ \\
\hline New pressure ulcer & $4 / 15(27)$ & $31 / 181(17)$ & $1.6(0.6-4.0)$ \\
\hline Any iatrogenic event & $20 / 81(25)$ & $15 / 115(13)$ & $1.9(1.0-3.4) \dagger$ \\
\hline \multicolumn{4}{|l|}{ Axis 4 , intercurrent illness } \\
\hline Malnutrition & $8 / 14(57)$ & $27 / 182(15)$ & $3.9(2.0-7.5) \dagger$ \\
\hline Respiratory insufficiency & $6 / 14(43)$ & $29 / 182(16)$ & $2.7(1.2-5.8) \dagger$ \\
\hline Intercurrent infection & $4 / 14(29)$ & $31 / 182(17)$ & $1.7(0.7-4.3)$ \\
\hline Dehydration & $23 / 110(21)$ & $12 / 86(14)$ & $1.5(0.8-2.8) \dagger$ \\
\hline
\end{tabular}

*Relative risks (RRs) in this table are the crude ratio of number of events (delirium) in the group when the precipitating factor is present vs when the factor is absent. $\mathrm{Cl}$ indicates confidence interval.

tIndicates 11 variables included in final stepwise binomial (relative risk) model.

rowed to five final variables. The five independent precipitating factors for delirium included in the final multivariable model (Table 3 ) were use of physical restraints, malnutrition, more than three medications added, use of bladder cath- eter, and any iatrogenic event. The adjusted RR derived from the model coefficients are estimates of the independent contribution of each variable to the risk of developing delirium, while controlling for the other variables. For example, the use of physical restraints is associated with a 4.4-fold increased risk of delirium, while controlling for all other variables in the model.

The "more than three medications added" variable was based on changes in the count of total number of types of medications received each day. Although data were not collected on each type of medication received, detailed information was available on 17 classes of psychoactive medications (including sedative-hypnotics, narcotics, anticholinergies, and $\mathrm{H}_{2-}$ blockers). Of the 107 patients who had more than three medications added, 75 $(70 \%)$ had at least one psychoactive medication added: $32(30 \%)$ had one psychoactive medication added, $21(20 \%)$ had two added, 14 (13\%) had three added, seven $(6 \%)$ had four added, and one $(1 \%)$ had five added.

The "malnutrition" variable was based on a serum albumin level of less than 30 $\mathrm{g} / \mathrm{L}$ occurring during hospitalization, but preceding the onset of delirium. This variable was associated with a mean weight loss of $5.6 \mathrm{~kg}$ during hospitalization, compared with a mean weight loss of $0.7 \mathrm{~kg}$ in those with normal nutritional status $(P=.03)$. For the 116 patients $(59 \%)$ who were missing serum albumin levels, we verified that a missing serum albumin level was not a risk factor for delirium (parameter estimate $=-0.57 ; P=.13$; the effect was actually protective), and that we were justified in combining the missing group with the group with serum albumin levels of $30 \mathrm{~g} / \mathrm{L}$ or greater.

\section{Performance of the Predictive Model}

Development Cohort.-We developed a risk stratification system (Table 4) by adding 1 point for each of the final five precipitating factors present. We combined groups with similar proportions of patients developing delirium to create three risk groups. The low-risk group included patients with no precipitating factors present, intermediate-risk group patients with one to two precipitating factors present, and the high-risk group patients with three or more precipitating factors present. The delirium rate by person for the low-, intermediate-, and high-risk groups were $3 \%, 20 \%$, and $59 \%$, respectively ( $\chi^{2}$ trend $=34.8$; $P<.001$ ), representing a 22.5 -fold increased delirium risk between low-risk and high-risk groups. The corresponding rates per 100 person-days (or percentage developing delirium per day) were $0.3 \%, 3.6 \%$, and $21.3 \%$, respectively $\left(\chi^{2}\right.$ trend $\left.=64.9 ; P<.001\right)$, representing a 71.7-fold increased delirium risk between low-risk and high-risk groups. The delirium rate of $21.3 \%$ per day corresponds to an $88.4 \%$ rate of delirium for a 9 -day hospital stay. 
Table 3.- Independent Precipitating Factors for Delirium in the Development Cohort $(n=196)^{*}$

\begin{tabular}{lc}
\hline \multicolumn{1}{c}{ Precipitating Factor } & $\begin{array}{c}\text { Adjusted RR } \\
(\mathbf{9 5 \%} \text { Cl) }\end{array}$ \\
\hline Use of physical restraints $(n=31)$ & $4.4(2.5-7.9)$ \\
Malnutrition $(n=14)$ & $4.0(2.2-7.4)$ \\
$>3$ Medications added $(n=18)$ & $2.9(1.6-5.4)$ \\
Use of bladder catheter $(n=50)$ & $2.4(1.2-4.7)$ \\
Any iatrogenic event $(n=81)$ & $1.9(1.1-3.2)$ \\
\hline
\end{tabular}

* $n$ indicates number of patients with the precipitating factor. Adjusted relative risks (RRs) were derived from binomial (relative risk) modeling. $\mathrm{Cl}$ indicates confidence interval.

Validation Cohort.-Of the 312 patients in the validation cohort, $47(15 \%)$ had new-onset delirium by hospital day 9 , with a median onset on hospital day 6 (range, 3 to 45 days). The median length of hospital stay for this cohort was 9 days (range, 2 to 79 days). The rates and times of onset of delirium and lengths of hospital stay did not differ significantly in the two cohorts. Although the two cohorts were comparable in most baseline characteristics (Table 1), the validation cohort had significantly fewer subjects living alone ( $28 \%$ vs $43 \%$ ) and somewhat higher mean APACHE II $^{23}$ scores (15.4 vs 14.0 ) at baseline.

Applying the risk stratification system to the validation cohort (Table 4), the delirium rates by person for the low-, intermediate-, and high-risk groups were $4 \%, 20 \%$, and $35 \%$, respectively $\left(\chi^{2}\right.$ trend $=24.8 ; P<.001)$, representing a ninefold increased delirium risk between lowrisk and high-risk groups. The corresponding rates per 100 person-days (or percentage developing delirium per day) were $0.5 \%, 3.3 \%$, and $8.2 \%$, respectively $\left(\chi^{2}\right.$ trend $=41.4 ; P<.001$ ), representing a 17.5-fold increased delirium risk between low-risk and high-risk groups. The delirium rate of $8.2 \%$ per day corresponds to a $53.7 \%$ rate of delirium for a 9-day hospital stay.

\section{Interrelationship of Baseline and Precipitating Factors}

Development Cohort.-The interrelationship of baseline and precipitating factors groups is presented in cross-stratified format in Figure 2. The values presented are delirium rates per 100 persondays (or percentage developing delirium per day). The values increase progressively from low-risk to high-risk groups in all directions, ie, across rows, across columns, or diagonally. This "double-gradient phenomenon" 29 indicates that both baseline and precipitating factors contribute to delirium in independent and substantive ways. When this double-gradient phenomenon was formally tested by fitting a binomial regression model that included baseline risk score, precipitating factors score, and hospital day, the contributions to the model of base-

Table 4.-Performance of the Predictive Model in the Two Cohorts*

\begin{tabular}{|c|c|c|c|c|c|}
\hline Risk Group & $\begin{array}{l}\text { No. of } \\
\text { Factors }\end{array}$ & $\begin{array}{c}\text { Delirium Rate } \\
\text { per Person, } \\
\text { No. (\%) }\end{array}$ & RR $(95 \%$ Cl) & $\begin{array}{c}\text { Delirium Rate } \\
\text { per Day, } \\
\text { No. (\% per Day)t }\end{array}$ & $\operatorname{RR}(95 \% \mathrm{Cl})$ \\
\hline \multicolumn{6}{|c|}{ Development Cohort $(n=196)$} \\
\hline Low & 0 & $2 / 76(3) \ddagger$ & 1.0 (Referent) & $2 / 673(0.3) \S$ & 1.0 (Referent) \\
\hline Intermediate & $1-2$ & 20/98 (20) & $7.8(2.5-24.5)$ & 20/559 (3.6) & $12.0(3.9-37.2)$ \\
\hline High & $\geq 3$ & $13 / 22(59)$ & $22.5(8.7-57.9)$ & $13 / 61(21.3)$ & $71.7(33.7-152.5)$ \\
\hline \multicolumn{6}{|c|}{ Validation Cohort $(n=312)$} \\
\hline Low & 0 & $5 / 125(4) \|$ & 1.0 (Referent) & $5 / 1063(0.5) \uparrow$ & 1.0 (Referent) \\
\hline Intermediate & $1-2$ & $31 / 156(20)$ & $5.0(2.2-11.0)$ & $31 / 934(3.3)$ & $7.1(3.2-15.7)$ \\
\hline High & $\geq 3$ & $11 / 31(35)$ & $8.9(3.9-20.3)$ & $11 / 134(8.2)$ & $17.5(8.1-37.4)$ \\
\hline
\end{tabular}

*Each patient's risk group was determined by adding 1 point for each precipitating factor present: use of restraints, urinary catheter, more than three medications added, any iatrogenic event, and malnutrition. RR indicates relative risk: $\mathrm{Cl}$, confidence interval for relative risk estimate.

tIndicates percentage of delirium per day or rate per 100 person-days.

$\neq x^{2}$ overall=37.8, $P<.001 ; x^{2}$ trend $=34.8, P<.001$.

$\S x^{2}$ overall $=96.5, P<.001 ; x^{2}$ trend $=64.9, P<.001$.

$\| x^{2}$ overall $=24.8, P<.001 ; \chi^{2}$ trend $=24.8, P<.001$.

ी $\chi^{2}$ overăll $=41.1, P<.001 ; \chi^{2}$ trend $=41.1, P<.001$.

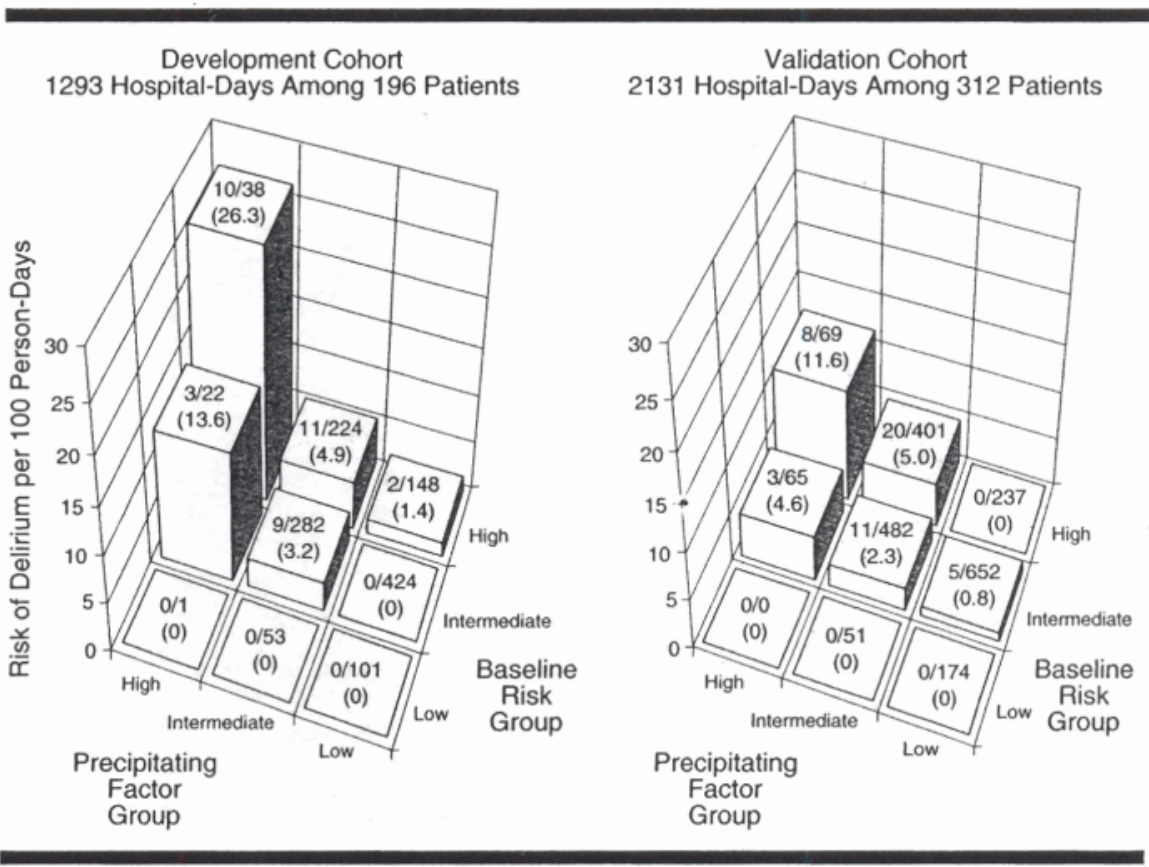

Figure 2.-Interrelationship of baseline and precipitating factors. Cross-stratification of baseline risk and precipitating factors groups. Rates are given as number of patients with delirium per 100 person-days (or percentage developing delirium per day). The double-gradient phenomenon ${ }^{29}$ is shown by the increasing risk of delirium when moving from low-risk to high-risk groups in all directions (across rows, columns, or diagonally). The delirium rates of $26.3 \%$ per day (development cohort) and $11.6 \%$ per day (validation cohort) correspond to rates of $99.9 \%$ and $67 \%$ for delirium, respectively, during a 9 -day hospital stay.

line and precipitating factors were documented to be independent and statistically significant (baseline risk score, $P<.05$; precipitating factors score, $P<.001)$.

The absence of delirium cases in the low baseline risk group confirms our hypothesis that these low-risk patients are resistant to the development of delirium. Conversely, patients at high baseline risk for delirium are vulnerable to delirium with any degree of precipitating factors.

When comparing our predictive models to the actual observed data, we obtained a model $\mathrm{G}^{2}$ value of $3.9(d f=5$; $P=.57)$. This value indicates that the modeled or expected values are similar to the observed values given in Figure 2 . Thus, the models perform well and correctly classify most cases.

Validation Cohort.-Although the validation cohort had lower rates of delirium at each risk level (Figure 2), the trends remain statistically significant and the double-gradient phenomenon clearly persists. All of the important findings cited herein are validated in this independent cohort.

The model $\mathrm{G}^{2}$ value for this cohort is $4.1(d f=5, P=.54)$, again demonstrating that the models perform well and supporting the findings from the development cohort. In fact, the models in development and validation cohorts behave 
in a markedly similar fashion, lending strong validation for this approach.

\section{COMMENT}

In this prospective study, five independent precipitating factors for delirium were identified: use of physical restraints, malnutrition, more than three medications added, use of bladder catheter, and any iatrogenic event. A simple predictive model based on the presence of these five factors can be used to predict which elderly medical patients are at high risk (and conversely, at low risk) for development of delirium during the first 9 days of hospitalization.

Strengths of the current study include the systematic assessment for delirium using a standardized, validated instrument and the prospective validation in a separate sample. Although there were some statistically significant differences in baseline characteristics between the two cohorts (Table 1), the predictive model did create a distinct and significant risk gradient in the validation cohort, with a 17.5fold increased risk for delirium between low- and high-risk groups.

Our findings lend strong support for our multifactorial model for delirium, demonstrating that both baseline (predisposing vulnerability) factors present on admission and precipitating (hospitalization-related) factors contribute to the development of delirium in important and independent ways. In addition, baseline

\section{References}

1. Inouye SK. The dilemma of delirium: clinical and research controversies regarding diagnosis and evaluation of delirium in hospitalized elderly medical patients. Am J Med. 1994;97:278-288.

2. Williams MA, Campbell EB, Raynor WJ, Musholt MA, Mlynarczyk SM, Crane LF. Predictors of acute confusional states in hospitalized elderly patients. Res Nurs Health. 1985;8:31-40.

3. Gustafson Y, Berggren D, Brannstron B, et al. Acute confusional states in elderly patients treated for femoral neck fracture. J Am Geriatr Soc. 1988; 36:525-530.

4. Foreman MD. Confusion in the hospitalized elderly: incidence, onset, and associated factors. Res Nurs Health. 1989;12:21-29.

5. Rockwood K. Acute confusion in elderly medical patients. J Am Geriatr Soc. 1989;37:150-154

6. Rogers MP, Liang MH, Daltroy LH, et al. Delirium after elective orthopedic surgery: risk factors and natural history. Int $J$ Psychiatry $\mathrm{Med}$. 1989;19:109-121.

7. Francis J, Martin D, Kapoor WN. A prospective study of delirium in hospitalized elderly. JAMA. 1990;263:1097-1101.

8. Schor JD, Levkoff SE, Lipsitz LA, et al. Risk factors for delirium in hospitalized elderly. JAMA. 1992;267:827-831.

9. Williams-Russo P, Urquhart RN, Sharrock MD, Charlson ME. Post-operative delirium: predictors and prognosis in elderly orthopedic patients. $J \mathrm{Am}$ Geriatr Soc. 1992;40:759-767.

10. Marcantonio ER, Goldman L, Mangione CM, et al. A clinical prediction rule for delirium after elective noneardiac surgery. JAMA. 1994;271:134-139. 11. Pompei P, Foreman M, Rudberg MA, Inouye SK, Braund V, Cassel CK. Delirium in hospitalized older persons: outcomes and predictors. J Am Geri- and precipitating factors are highly interrelated and contribute to delirium in a cumulative fashion. The identification of a group at extremely low risk for delirium (the low baseline risk, low precipitating factors group) is another noteworthy contribution of this study.

An important limitation of the study is the fact that delirium assessments were done every other day. However, these assessments were supplemented by interviews with nursing staff and daily nursing observations and medical record reviews. Through these methods, we believe that all clinically important cases of delirium were identified. The delirium ratesin our cohorts (18\% and $15 \%$ ), which were restricted to the first 9 days of hospitalization, were comparable with those of previous studies that have examined new-onset delirium in medical patients. $5,7,11,30,31$

Although not specifically identified in previous predictive models, ${ }^{2-11}$ the five precipitating factors for delirium identified in the current study are supported by the previous delirium literature, as well as by a priori clinical relevance and expert opinion. Physical restraints, which preceded the onset of delirium by at least 24 hours, were most often used for fall prevention and contributed to involuntary immobilization. Similarly, the use of indwelling bladder catheters contributed to involuntary immobilization, as well as predisposing to urinary tract infections.

atr Soc. 1994;42:809-815

12. Tinetti ME, Inouye SK, Gill TM, Doucette JT Shared risk factors for falls, incontinence, and functional dependence: unifying the approach to geriatric syndromes. JAMA. 1995;273:1348-1353.

13. Inouye SK, Viscoli CM, Horwitz RI, Hurst LD, Tinetti ME. A predictive model for delirium in hospitalized elderly medical patients based on admission characteristics. Ann Intern Med. 1993;119:474-481.

14. Katz S, Ford AB, Moskowitz RW, Jackson BA Jaffe MW. Studies of illness in the aged: the index of ADL, a standardized measure of biological and psychosocial function. JAMA. 1963;185:914-919.

15. Folstein MF, Folstein SE, McHugh PR. The Fol stein Mini-Mental State Examination: a practical method for grading the cognitive state of patients for the clinician. J Psychiatr Res. 1975;12:189-198.

16. Ventry IM, Weinstein B. The Hearing Handicap Inventory for the elderly: a new tool. Ear Hear 1982;3:128-134.

17. Beck S, Brook RH, Lohr KN, Goldberg GA. Conceptualization and Measurement of Physiologic Health for Adults: Hearing Loss. Santa Monica, Calif: RAND; 1981;14:72-75. RAND publication RAND/R-2262-14-HHS.

18. Inouye SK, van Dyck CH, Alessi CA, Balkin S, Siegal AP, Horwitz RI. Clarifying confusion: the Confusion Assessment Method: a new method for detection of delirium. Ann Intern Med. 1990;113:941-948. 19. Inouye SK, Wagner DR, Acampora D, et al. A predictive index for functional decline in hospitalized elderly medical patients. $J$ Gen Intern Med. 1993;8:645-652.

20. Charlson ME, Sax FL, MacKenzie CR, Fields SD, Braham RL, Douglas RG. Assessing illness severity: does clinical judgment work? J Chronic Dis. 1986;39:439-452.
The "more than three medications added" variable was associated with addition of psychoactive medications in $70 \%$ of patients; however, this high degree of flux in medications may also have indicated multiple medically active problems. Finally, any iatrogenic event and malnutrition were associated with an increased risk for delirium.

Most importantly, all five of the identified precipitating factors are modifiable and amenable to intervention. Future studies will be needed to test the effectiveness of intervention strategies designed to address these precipitating factors. Used in combination, the baseline risk and precipitating factors models provide a sound basis with which to target at-risk patients and design interventions for much needed clinical trials for prevention of delirium in hospitalized elderly patients.

This study was supported in part by grants from the Commonwealth Fund (grant 94-90), the Retirement Research Foundation (grants 91-66 and 94-71), and a pilot study grant by the Claude D. Pepper Older Americans Independence Center (P60AG10469) from the National Institute on Aging. Dr Inouye is recipient of Academic Award K08AB00524 from the National Institute on Aging.

We thank the patients, families, and nurses at Yale-New Haven Hospital who participated in the study; Máry Tinetti, MD, and Christianna Williams, MPH, for helpful review of the manuscript; and Rob- bin Bonanno and Geraldine Hawthorne for preparation of the manuscript. This work is dedicated to the memory of Joshua Bryan Inouye Helfand.

21. Blessed G, Tomlinson BE, Roth $\mathrm{M}$. The association between quantitative measures of dementia and of senile change in the cerebral grey matter of elderly subjects. Br J Psychiatry. 1968;114:797-811. 22. Uhlmann RF, Larson EB, Buchner DM. Correlations of Mini-Mental State and Modified Dementia Rating Scale to measures of transitional health status in dementia. $J$ Gerontol. 1987;42:33-36.

23. Knaus WA, Draper EA, Wagner DP, Zimmerman JE. APACHE II: a severity of disease classification system. Crit Care Med. 1985;13:818-829. 24. Becker PM, McVey LJ, Saltz CC, Feussner JR, Cohen HJ. Hospital-acquired complications in a randomized controlled clinical trial of a geriatric consultation team. JAMA. 1987;257:2313-2317.

25. Steel K, Gertman PM, Crescenzi C, Anderson J. Iatrogenic illness on a general medical service at a university hospital. N Engl J Med. 1981;304:638-642. 26. Wacholder S. Binomial regression in GLIM: estimating risk ratios and risk differences. $\mathrm{Am} J$ Epidemiol. 1986;123:174-184.

27. Hosmer DW, Lemeshow S. Applied Logistic Regression. New York, NY: John Wiley \& Sons Inc; 1989:131-133.

28. McCullagh P, Nelder JA. Generalized Linear Models: Monographs on Statistics and Applied Probability. New York, NY: Chapman \& Hall; 1983: 79-86.

29. Feinstein AR. Clinimetrics. New Haven, Conn: Yale University Press; 1987:57-88.

30. Rosin AJ, Boyd RV. Complications of illness in geriatric patients in hospital. J Chronic Dis. 1966; 19:307-313.

31. Hodkinson HM. Mental impairment in the elderly. J R Coll Physicians Lond. 1973;7:305-317. 